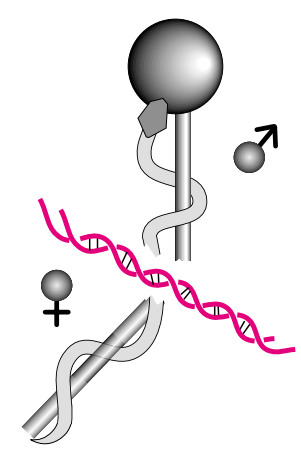

\section{Avis du Comité consultatif national d'éthique pour les sciences de la vie et de la santé (France)}

La génétique, science de la transmission des caractères héréditaires, créée par Gregor Mendel en 1865, a déjà une histoire tumultueuse puisqu'elle a servi, dans la première moitié de ce siècle, de caution scientifique au courant eugénique très puissant dans différents pays.

C'est que son objet même la situe au cour d'un des questionnements constitutifs de la personne humaine: d'où viens-je, qui suis-je, que vais-je transmettre à mes descendants, en quoi suis-je tout à la fois semblable aux autres personnes et différent d'elles? Cela explique que la science génétique ait eu et puisse avoir plus de répercussions individuelles, politiques et sociales qu'aucune autre science.

Aujourd'hui, les progrès de la recherche en génétique humaine permettent d'entrevoir le jour prochain où tous les gènes humains, au nombre d'environ 100000 , auront été identifiés, localisés sur les chromosomes, et où leur fonction, ou au moins leur implication dans des maladies génétiques, sera à peu près connue. Et le mythe du gène, support du programme de la vie, est tel que cela conduit à l'illusion qu'une connaissance parfaite du génome d'un individu donnerait accès à la réalité et au destin de la personne. C'est cette image à laquelle renvoie une métaphore comme celle du grand livre de la vie dont il suffirait de connaître l'alphabet et la syntaxe génétiques pour parvenir à l'essence de l'être. Or une telle conception est scienti- fiquement inacceptable et éthiquement dangereuse.

Un gène correspond à un facteur de détermination biologique dont l'expression et la signification fonctionnelle dépendent souvent de l'influence des autres gènes et de l'environnement, lui-même imprévisible à long terme. Même si on parvenait à la connaissance de la signification de tous les gènes et de la combinatoire de leur expression, on ne connaîtrait que les propriétés biologiques des cellules vivantes et des organismes, et non l'univers psychique et affectif de la personne humaine. La propriété génétiquement déterminée permettant d'accéder aux capacités psychiques de l'être humain est, en effet, d'une particulière malléabilité à l'environnement et aux empreintes d'un monde spécifiquement humain et extérieur aux déterminismes génétiques: celui de la culture, des connaissances, des pratiques sociales, des valeurs, de l'histoire des sociétés humaines, elles-mêmes modulées par la personnalité propre de ceux par qui elles sont transmises, par les événements de la vie, les expériences affectives, les épreuves... La personne humaine est, par conséquent, irréductible à cet élément de son corps, le génome. Il n'empêche que, même limité à la possibilité de prédire, plus ou moins exactement, plus ou moins précisément, certains aspects du destin biologique des êtres humains - susceptibilité à des maladies ou autres caractéristiques non pathologiques -, le pouvoir issu de la génétique est de Saint-Dominique, 75007 Paris, France. 
nature à avoir de profondes répercussions pour les individus et la société, aujourd'hui et dans le futur.

Les progrès dans les connaissances en génétique humaine et les avancées techniques dans les méthodes de diagnostic, en particulier celles qui relèvent de la génétique moléculaire, apportent de nouveaux outils qui ont constitué petit à petit les bases d'une médecine de prévision, dont on espère qu'elle ouvrira la voie à la prévention. En effet, un vaste champ d'applications médicales, qui dépasse de beaucoup l'étude des maladies héréditaires, s'ouvre aux examens des caractéristiques génétiques. Il devrait encore s'élargir dans les prochaines années grâce aux recherches sur le génome humain. C'est ainsi qu'il devient possible d'envisager d'étendre les politiques de prévention, depuis la prévention des handicaps à la naissance jusqu'à la prévention d'affections de l'adolescent et de l'adulte (diabète, cancers, maladies cardio-vasculaires), voire du vieillard (maladie d'Alzheimer).

Le principe de base de la médecine de prévision est de prévoir l'apparition de certaines maladies avant l'expression de leurs symptômes. Mais de grandes incertitudes existent sur la valeur des prévisions, sur la possibilité réelle d'empêcher ces maladies et sur le bénéfice pour l'individu et la société de cette forme de prévention.

La prévention vise à empêcher ou à retarder l'apparition des maladies et représente a priori une conduite tout à fait souhaitable. Son intérêt s'est révélé indiscutable dans de nombreux domaines: par exemple, la lutte contre des maladies infectieuses grâce à la vaccination, ou les politiques en périnatalogie qui ont conduit à des réductions spectaculaires de la morbidité et de la mortalité infantile.

La prévention peut prendre des formes extrêmement diverses et qui ne sont pas nécessairement médicales, comme les campagnes d'information sur les dangers du tabagisme ou de la circulation routière, pour ne citer que quelques exemples. Elle peut s'appliquer à la totalité de la po- des groupes de sujets soumis, par exemple, à un même facteur de risque lié à l'environnement, comme les travailleurs exposés à des risques professionnels, ou, enfin, à des individus et à des familles que leurs caractéristiques de comportement, d'environnement, biologiques ou génétiques désignent comme plus particulièrement susceptibles de développer une maladie ou de la transmettre à leur descendance. Dans les deux premières situations, la prévention peut être qualifiée de « collective» et est décidée indépendamment de l'état du sujet, alors que, dans la troisième, elle est «individuelle» et dépend des caractéristiques du sujet. La réflexion éthique n'est pas identique pour les trois situations.

L'expérience des campagnes de santé publique (tabagisme, accidents de la route) montre clairement les difficultés à observer les mesures préventives préconisées lorsqu'il s'agit de modifier des comportements socialement valorisés, même lorsque la souffrance et la mort sont prévisibles. Ces problèmes pourraient devenir considérables en cas de multiplication des contraintes associées à la mise en évidence d'un nombre de plus en plus important de facteurs de susceptibilité génétiquement déterminés.

Il est donc indispensable de procéder à l'évaluation des préventions en tenant compte - en plus des critères scientifiques et médicaux habituels de bénéfice et d'effets néfastes - des phénomènes de contraintes et de résistances psychologiques et sociales qui entrent en jeu lorsqu'on tente de promouvoir de nouvelles règles de conduite. Les enjeux économiques, où seront en compétition des espoirs d'économie pour le budget de la Santé et des objectifs de marché pour les industries de santé et le corps médical, doivent également être pris en considération.

On devra aussi s'interroger sur la pertinence d'une approche de la prévention qui se fonderait quasi exclusivement sur l'apport de la génétique, alors que les facteurs génétiques ne sont pas les seuls en cause dans le déclenchement de nombreuses affections et que la préven- tion exigerait également des mesures prises au niveau de l'environnement. Les examens des caractéristiques génétiques présentent des particularités sur lesquelles il convient d'insister: ces caractéristiques sont à la fois des éléments constitutifs de l'individu en tant qu'être unique et des éléments qui relient l'individu à sa famille, passée, présente et à venir. Leur examen et leur révélation touchent donc l'individu dans sa nature biologique intime et dans ses liens avec sa famille. L'analyse éthique doit tenir compte de ce double rapport de l'individu à lui-même et à sa famille. La mise en évidence d'un caractère génétique peut être ressentie comme une a-normalité, voire comme une discrimination et une stigmatisation de l'individu, qui met en jeu la responsabilité éthique de la société.

Cela amène à se poser la question, difficile, de la destinée et de la liberté confrontées à la connaissance des prédispositions génétiques. D'un côté, l'ignorance est rarement facteur de liberté, et la connaissance de sa susceptibilité à des affections évitables rend l'individu responsable d'en tirer les conséquences. Il est, après tout, admis par tous que c'est en assumant son destin que l'on peut exercer au mieux sa réelle liberté, qui ne serait sans cela que velléité. Il n'empêche que l'on ne peut manquer de s'interroger sur la signification réelle de l'exercice de sa liberté par une personne à laquelle ses prédispositions génétiques ne laissent que le choix entre une existence parfois terriblement contrainte ou des mutilations à visée préventive, et le risque d'une maladie incurable. Une autre dimension individuelle du destin génétique dévoilé est qu'il équivaut parfois, en cas de transmission d'une maladie grave, à une malédiction sur le lignage, les parents étant coupables d'avoir transmis le mauvais gène à leurs enfants, à leur tour, atteints par le sentiment de culpabilité de pouvoir le transmettre à leur descendance.

D'autres questions relèvent de règles éthiques générales s'appliquant en médecine: en matière de prévision et de prévention, avant de prescrire et de réaliser un examen chez des su- 
jets en bonne santé et qui souvent ne sont pas demandeurs, il faut avoir envisagé et évalué les conduites médicales préventives et curatives qui pourront être mises en œuvre en fonction des informations obtenues grâce à ces examens.

Une appréciation éthique de la prévention doit tenir compte de l'évaluation des bénéfices dont la perspective doit surpasser nettement celle des effets nocifs. La prévention comporte, dans un premier temps, une évaluation d'un risque. Cette évaluation peut se faire, soit chez un individu, soit dans une famille, soit dans un groupe ethnique, soit dans l'ensemble de la population.

Il faut bien distinguer:

- Les diagnostics présymptomatiques qui mettent en évidence chez un individu l'existence de l'anomalie génétique avant les manifestations cliniques qui peuvent en résulter. Ces diagnostics concernent des maladies génétiques dont le risque de survenue chez les sujets porteurs de la mutation est très élevé. Ils peuvent être réalisés dans la période prénatale, néonatale ou au cours de la vie.

En ce domaine, on peut schématiquement opposer les situations où une prévention déjà évaluée est possible aux situations où il n'existe pas de prévention possible. Par exemple, le diagnostic présymptomatique d'une mutation responsable de la forme familiale de la polypose recto-colique permet la mise en œuvre d'une surveillance préventive et d'interventions curatives. Le diagnostic présymptomatique de la mutation responsable de la maladie de Huntington ne permet qu'une information sur un risque certain d'être atteint par cette maladie, sans pouvoir proposer une conduite préventive et curative.

- Les diagnostics génétiques qui ont pour objectif d'évaluer le risque pour la descendance de l'individu testé. Ces diagnostics étant pratiqués, soit dans le cadre d'études familiales, soit dans un cadre plus général de dépistages dans la population.

Par exemple, dans certaines régions méditerranéennes, la forte fréquence de la $\beta$-thalassémie (anémie méditerranéenne) conduit à proposer le dépistage, vers l'âge de vingt ans, des sujets hétérozygotes pour la mutation, en vue d'informer les couples $\mathrm{du}$ risque pour leur descendance et des possibilités d'un diagnostic prénatal.

- Les diagnostics probabilistes de prédisposition à une maladie grave qui ont pour objectif d'évaluer chez un individu le risque de survenue de l'affection, en comparaison de ce risque dans la population générale. Dans ce cas également, il faudra distinguer les situations avec ou sans prévention possible et évaluée.

S'il est clair que, dans une maladie monogénique, un gène muté est la cause première des troubles, dans une maladie multifactorielle, les différents facteurs de risque, génétiques ou liés à l'environnement, appartiennent à une chaîne plus ou moins longue d'événements. Cela signifie qu'à l'extrémité de cette chaîne la détermination éventuelle d'une maladie (ou plus généralement d'un «phénotype» observable) est souvent un effet cumulé des différents éléments de cette chaîne. Si des mesures de prévention étaient possibles, elles ne pourraient s'exercer que sur des facteurs extérieurs dont on aurait alors montré l'interaction avec les facteurs génétiques.

La perspective de ces répercussions personnelles et sociales appelle de toute évidence une réflexion éthique. De fait, cette réflexion a pris, en France comme dans le monde, un nouvel essor depuis plus de dix ans en même temps que se développait la génétique moléculaire. Nombreuses sont ses expressions, non seulement sous forme de questions mais de directives, orientations ou parfois règles de droit concernant la connaissance du génome et ses applications. Le développement actuel rapide des tests génétiques - dont l'efficacité précédera sans doute assez longtemps celle des thérapeutiques correspondantes - invite à rassembler les résultats de ces réflexions éthiques, à les actualiser et à les préciser, notamment pour une application dans le cadre législatif que la France s'est donnée en juillet 1994.

\section{Recommandations}

1. L'examen des caractéristiques génétiques d'un individu peut avoir, quel que soit son résultat, de profondes répercussions sur la vie du sujet qui s'y prête. Le respect de son autonomie exige qu'il ait une compréhension aussi complète que possible des conséquences de sa décision de se soumettre ou non à cet examen.

Cette compréhension implique une information sur la nature de l'examen, la signification des résultats, l'existence éventuelle d'une prévention et d'une thérapie ainsi que leurs contraintes. Cette information doit être donnée par un professionnel ayant une bonne connaissance de la génétique médicale et être directe et orale pour permettre un dialogue, puis consignée dans un document écrit.

Toute détermination de caractères du génotype d'un individu ne doit être entreprise qu'à des fins médicales sur prescription ou à des fins scientifiques et que si le sujet a donné spécifiquement son consentement écrit.

Les résultats de l'examen doivent être communiqués directement par un médecin, qui, de par sa compétence, pourra expliquer aux sujets testés la signification de ces résultats. Un suivi du sujet testé devra être assuré, pour pallier les éventuelles répercussions psychologiques du résultat, que celui-ci soit positif ou négatif. Certaines informations peuvent avoir un effet potentiellement néfaste pour l'individu. Celui-ci peut donc refuser de connaître les résultats de l'examen et son droit de ne pas savoir doit toujours être respecté.

2. Le secret médical doit être respecté vis-à-vis des tiers, y compris les autres membres de la famille. Lorsque la découverte d'une anomalie génétique de caractère familial conduit à envisager un prélèvement biologique sur l'ensemble des membres de la famille, ceux-ci devront être sollicités directement par le sujet demandeur et non par le médecin. Si le sujet refuse de faire connaître aux membres de sa famille le risque révélé par l'examen génétique qu'il a 
subi, le médecin sera dans l'impossibilité de les prévenir du risque éventuel qu'ils ont de développer une maladie ou de la transmettre à leur descendance. Le médecin devra informer le sujet testé de sa responsabilité et tout faire pour le convaincre d'informer ses proches. En cas d'échec, le devoir de confidentialité et le secret médical seront en contradiction avec le devoir d'informer les sujets et les familles d'un risque qui peut être l'objet d'une prévention. Le médecin sera confronté à un grave conflit éthique sur lequel la société devra se prononcer, en tenant compte du caractère inacceptable de la non-assistance à personne en danger, particulièrement lorsque des enfants sont concernés.

L'examen des caractéristiques génétiques chez des enfants ne doit pas être envisagé comme une routine, mais doit toujours répondre à des situations particulières fondées sur une analyse des données médicales et des données familiales. Les parents et le médecin traitant ne doivent demander un examen pour l'enfant que si la maladie liée à son génotype peut se déclarer avant dixhuit ans ou peut bénéficier de mesures préventives instaurées avant dix-huit ans. L'enfant qui a subi un examen et doit faire l'objet, par la suite, d'une surveillance doit être informé dès qu'il sera apte à comprendre ces mesures.

Dans le cas où l'examen conduirait à évaluer un risque pour la future descendance de l'enfant, sa famille a un devoir de l'informer dès qu'il atteindra l'âge de procréer et qu'il sera apte à comprendre et à décider luimême de faire ces examens.

La transmission d'une génération à la suivante des informations relatives aux caractéristiques génétiques peut être nécessaire. Il convient de prévoir comment conserver les données génétiques familiales pendant au moins une génération et comment faire bénéficier les sujets à risque de cette information, quand elle leur sera utile.

3. L'informatisation des données nominatives relatives aux personnes qui ont fait l'objet d'un prélèvement doit être entreprise dans le respect de leur vie privée, conformément aux dispositions légales et aux recommandations émises par le CCNE dans ses précédents avis.

La conservation des échantillons biologiques qui pourraient être ultérieurement nécessaires à la mise en ouvre de tests diagnostiques ou de contrôle doit être assurée pour répondre aux demandes des personnes testées.

En cas d'extension des recherches à un domaine qui n'était pas prévu au moment du prélèvement, un nouveau consentement doit être demandé.

Lorsque les collections d'échantillons biologiques sont constituées dans le cadre d'un programme de recherche, les instigateurs de la recherche ont le devoir de mener cette recherche avec les moyens disponibles et dans les conditions indiquées au moment du consentement des individus qui ont fait l'objet d'un prélèvement.

La non-utilisation prolongée des collections par des chercheurs ne parvenant pas à progresser dans leur recherche pourrait être contraire à l'attente légitime des personnes qui ont consenti à l'utilisation des échantillons de leur ADN. Il faudrait donc établir des délais raisonnables audelà desquels l'accès aux collections serait ouvert à d'autres équipes pour travailler sur le programme auquel les personnes ont consenti. Dans le cas où les investigateurs renonceraient à mener eux-mêmes la recherche, ils devraient informer les personnes des modifications découlant de ce renoncement.

4. L'utilisation des résultats d'un examen des caractéristiques génétiques, à des fins autres que médicales ou de recherche, par exemple dans le cadre d'un contrat d'assurance ou d'emploi, est interdite, même si elle est le fait des sujets testés eux-mêmes ou qu'elle se fait avec leur accord.

Les cas où l'examen des caractéristiques génétiques peut être utile pour prévenir une maladie professionnelle sont rares dans l'état actuel des connaissances. L'utilisation des tests génétiques dans le cadre de la médecine du travail doit donc être exceptionnelle et strictement réser- vée à des cas limitativement énumérés, pour lesquels le risque pour l'individu est suffisamment bien établi et ne peut être supprimé par l'aménagement de l'environnement du travail.

5. Il convient de mettre en place les procédures d'agrément par l'Agence du médicament pour les réactifs utilisés dans les tests génétiques et de définir les conditions de leur commercialisation, ainsi que les procédures d'agrément et de contrôle des laboratoires pratiquant ces tests.

Lorsque les examens sont destinés à être effectués sur un grand nombre de sujets, des études préliminaires précises doivent être réalisées pour évaluer la valeur prédictive des tests et la valeur des mesures préventives et curatives qui vont être recommandées à la population sélectionnée par ces tests, cela avant l'obtention des agréments.

L'évaluation ne doit pas être seulement fondée sur des critères médicaux, mais elle doit également tenir compte de différents aspects de la qualité de la vie qui peut être affectée lors du dépistage lui-même ou en raison des contraintes liées à la prévention.

L'évaluation d'un programme de dépistage génétique et de prévention doit tenir compte du fait qu'un tel programme ne pourra être efficace que si le protocole envisagé est accepté par la population à laquelle il est destiné et par le corps médical.

6. Les attitudes des individus et des familles vis-à-vis des dépistages génétiques et des préventions sont liées à la qualité de l'information médicale donnée aux sujets concernés. Il est donc indispensable d'assurer une formation du personnel de santé en génétique médicale dans le cadre des cursus universitaires et de la formation continue des praticiens en exercice.

Une information pédagogique dispensée dès l'enseignement secondaire dans le cadre de l'enseignement de la biologie et de la philosophie devrait permettre de réduire les risques de discrimination ou de stigmatisation liés à la connaissance des caractéristiques génétiques. 
Les associations représentant les familles concernées par une maladie génétique doivent être encouragées dans leurs activités de diffusion d'informations médicales et scientifiques.

Il faut veiller à la qualité de l'information d'actualité destinée au public et qui, dans la recherche du «sensationnel», peut être à l'origine de faux espoirs. Il faut également veiller à ce que les intérêts financiers suscités par le marché potentiel considérable des tests génétiques ne portent atteinte à la loyauté et à l'indépendance de l'information.

7. Les tests génétiques apportent des informations sur l'identité des personnes et soulignent leur diversité qui contribue à la richesse de l'humanité. L'utilisation de ces informations à des fins de sélection ou de discrimination dans la vie sociale et économique, que ce soit dans le domaine des politiques de santé, de l'emploi ou des systèmes d'assurance, conduirait à franchir une étape d'une extrême gravité vers la mise en cause des principes d'égalité en droits et en dignité, et de solidarité entre tous les êtres humains, sur lesquels repose notre société. Le CCNE insiste sur la nécessité de respecter ces principes fondamentaux, quelle que soit la finalité de l'utilisation des tests génétiques. Il y va des Droits de l'Homme

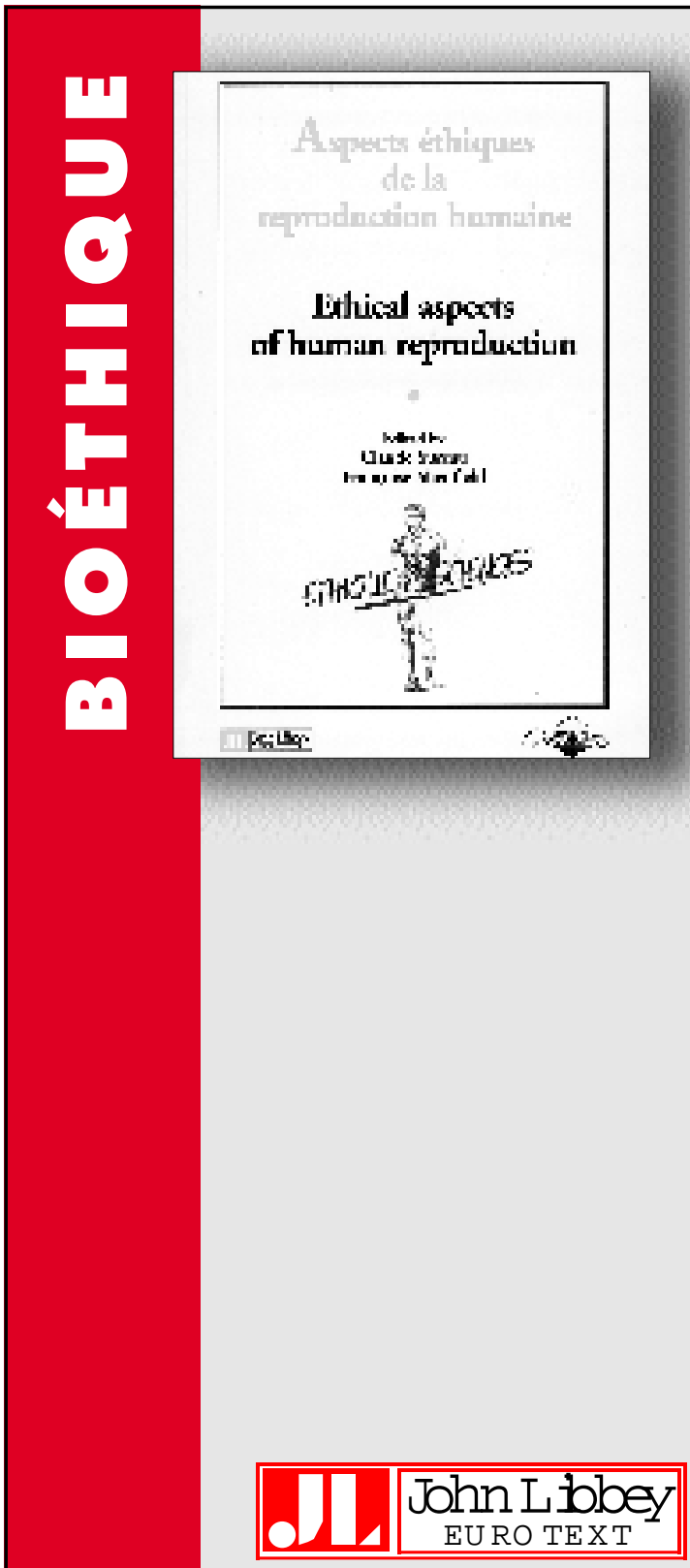

Éditions John Libbey Eurotext :

127 , avenue de la

République

92120 Montrouge - France

Tél. : 33 (1) 46730660

Fax : 33 (1) 40840999 